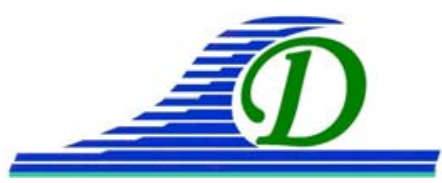

XIII ${ }^{\text {èmes }}$ Journées Nationales Génie Côtier - Génie Civil

Dunkerque, 2-4 juillet 2014

DOI:10.5150/jngcgc.2014.052 @ Editions Paralia CFL

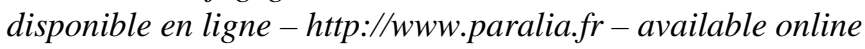

\title{
Modélisation de la dynamique du littoral de La Marsa- Gammarth à l'aide de NEMOS-GENESIS
}

\author{
Raghda MESTIRI ${ }^{1}$, Samira RAÏS ${ }^{1}$
}

1. Ecole Nationale d’Ingénieurs de Tunis, B.P. 37, Le Belvédère, 1002 Tunis, Tunisie. mestiri.raghda@gmail.com ; samira.rais@enit.rnu.tn

\section{Résumé :}

La côte tunisienne est exposée aux vagues de la mer Méditerranée. La région de la côte Nord-Est est l'une des zones les plus vulnérables à l'érosion côtière. Ce phénomène est particulièrement prononcé au niveau de la plage de Gammarth-La Marsa.

Cette étude présente une contribution à la conception d'une protection du rivage en utilisant la modélisation numérique de l'évolution littorale.

Cette modélisation est réalisée à l'aide du système NEMOS qui est constitué d'un ensemble de codes capables de simuler l'évolution à long terme de la forme en plan de la plage (code GENESIS) suivant les conditions imposées par la propagation de l'énergie spectrale des vagues (code STWAVE), la morphologie côtière et les facteurs anthropiques.

Le modèle d'évolution du littoral a d'abord été calibré à la situation de référence (position du trait de côte de 2000), ce qui a permis la détermination des valeurs adéquates des paramètres de calage, puis testé à la sensibilité des données d’entrée du modèle et enfin utilisé pour étudier les scénarios futurs. Il s'agit en premier lieu de simuler l'évolution naturelle de la côte de la zone étudiée. En effet, le module GENESIS nous a permis de prédire la position d'équilibre qui sera atteinte à l'horizon de 2030 en l'absence d'intervention dans le but de déterminer les zones les plus vulnérables à l'érosion et de proposer par la suite différents scénarios d'aménagement pour la protection de ces zones.

Mots clés : Modélisation, Houle, Erosion, Plage, Protection littorale, GENESIS.

Abstract: The Tunisian coast is exposed to the waves of the Mediterranean Sea. The region of the North-East coast is one of the most vulnerable coastal areas to erosion. This phenomenon is particularly pronounced at the beach of Gammarth-La Marsa.

This study presents a contribution to the design of a shoreline protection using numerical modeling of coastal evolution.

This modeling is performed using the NEMOS system which consists of a set of codes, able of simulating the long-term evolution of the beach (GENESIS code) according to the conditions imposed by the spread of energy spectral waves (STWAVE code), coastal morphology and anthropogenic factors. 


\section{Thème 2 - Dynamique sédimentaire}

The model of coastal evolution was first calibrated to the reference situation from the actual initial position, allowing the determination of appropriate values of the calibration parameters and then tested the sensitivity of the model input data and finally used to study future scenarios. It consists firstly to simulate the natural evolution of the coast of the study area. In fact, the GENESIS module has allowed us to predict the equilibrium position which will be reached by the year 2030 in order to determine the most vulnerable zones to erosion and to propose different management scenarios for the protection of these areas.

Keywords: Modeling, Swell, Erosion, Beach, Coastal Protection, GENESIS.

\section{Introduction}

La variation de l'équilibre sédimentaire du milieu côtier est mise en évidence par l'évolution du trait de côte. Elle est due essentiellement aux agents naturels et anthropiques auxquels la côte est constamment soumise, d'où l'importance de prendre des mesures préventives contre l'érosion des côtes sableuses et, si nécessaire, de réaliser des ouvrages de protection du littoral.

L'efficacité de ces mesures nécessite des études de protection des côtes qui passent nécessairement par l'examen des processus hydrodynamiques, hydrosédimentaires et l'influence anthropique affectant la morphodynamique des plages sableuses, ainsi que des études pour la prévention des impacts négatifs de ces ouvrages sur l'évolution de la plage et du trait de côte. Cette étude vise, d'une part, à évaluer l'équilibre sédimentaire de la zone en question à partir de l'étude de ses caractéristiques hydrodynamiques et propriétés sédimentaires et, d'autre part, à tenter de prédire son évolution et l'impact des ouvrages de protection projetés sur la stabilité du littoral en utilisant un modèle numérique.

\section{Site de l'étude}

La zone traitée dans le cadre de cette étude se situe sur la façade ouest du littoral nord du Golfe de Tunis comme le montre la figure 1. Il s'agit d'un tronçon de $6000 \mathrm{~m}$ appartenant à la côte de Gammarth-La Marsa.

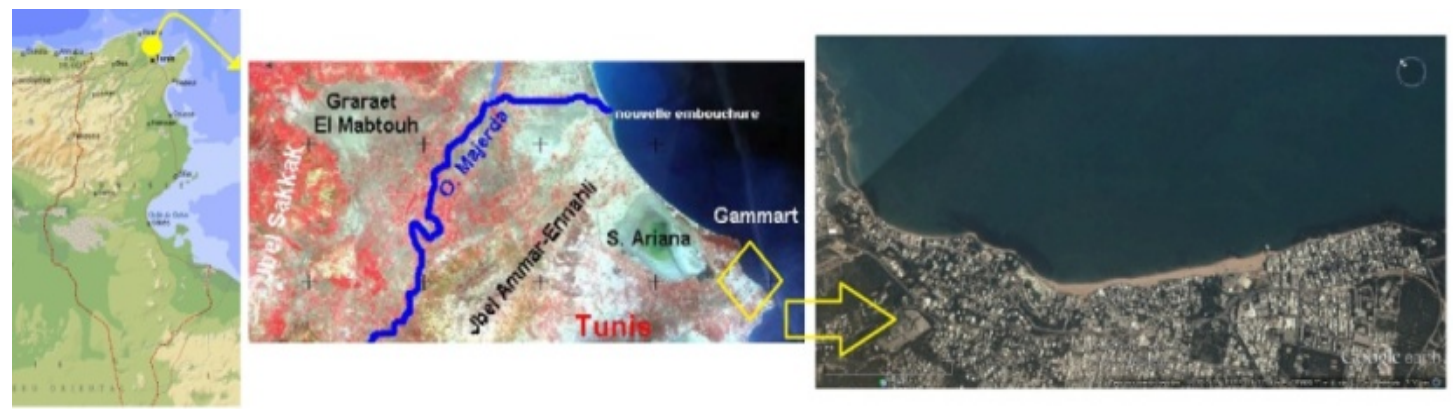

Figure 1. Situation géographique de la zone d'étude. 


\section{XIII ${ }^{\text {èmes }}$ Journées Nationales Génie Côtier - Génie Civil \\ Dunkerque, 2-4 juillet 2014}

La zone d'étude est caractérisée par une géomorphologie variable. Au nord, on repère le Cap Gammarth marqué par des falaises et des plages très restreintes. Au sud, le littoral est caractérisé par des espaces sableux plus développés, puis ces espaces se rétrécissent en se rapprochant de la colline de Sidi Bou Saïd qui représente la frontière sud du domaine de l'étude. Depuis environ un demi-siècle, cette côte subit une érosion continue par manque de disponibilité de sable en amont-transit et au niveau de Gammarth (la partie nord-ouest, zone concave) un recul moyen de 0.5 à 1.0 m/an est constaté. Sur le secteur de La Marsa, les évolutions sont plus faibles (recul du trait de côte de $0.5 \mathrm{~m} / \mathrm{an}$ ) (APAL, 2003). Sur l'ensemble du littoral et au niveau des zones sableuses, le sable constituant le littoral et les petits fonds est classé de sable fin à moyen.

\section{Description du modèle numérique}

\subsection{Présentation de NEMOS}

NEMOS "Nearshore Evolution MOdeling System" est un système comportant un ensemble de codes capables de simuler l'évolution à long terme de la forme en plan de la plage suivant les conditions imposées par les enregistrements de houle, la morphologie côtière, et les facteurs anthropiques tels que les structures littorales et la recharge artificielle de la plage.

Le système se compose de deux modules clés qui simulent la propagation de la houle STWAVE ("STeady State spectral WAVE") et le changement de la ligne de côte GENESIS ("GENEralized model for SImulating Shoreline change").

Il existe aussi des modules auxiliaires assurant la génération du maillage du domaine à partir des données bathymétriques GRIDGEN ("GRId GENerator"), la filtration des données WWWL ("Waves, Winds, Water Levels"), l'analyse statistique d'une série d'événements de houle WSAV ("Wave Station Analysis and Visualization"), et la création et la visualisation des spectres directionnels 2D en fonction de la fréquence et de l'angle de propagation de la houle SPECGEN ("SPECtrum GENerator").

Le module GENESIS assure la modélisation numérique de l'évolution de la position du trait de rivage, celui-ci est du type "one-line" (HANSON, 1988). La position du trait de côte en fonction du temps $t$, est régie par l'équation de la conservation de la masse suivante :

$$
\frac{\partial y}{\partial t}+\frac{1}{\left(h_{c}+d_{b}\right)}\left(\frac{\partial Q}{\partial x}+q\right)=0
$$

où $y$ : coordonnée vers l'offshore du trait de côte,

$h_{c}$ : profondeur de fermeture,

$d_{b}$ : hauteur moyenne de la berme,

$q$ : débits solides ponctuels (en $\mathrm{m}^{3} / \mathrm{m} / \mathrm{s}$ ) apportés (positifs) ou extraits (négatifs),

$x$ : coordonnée longitudinale (longshore). 


\section{Thème 2 - Dynamique sédimentaire}

La formule empirique de prédiction du transit littoral $Q\left(\mathrm{~m}^{3} / \mathrm{s}\right)$ utilisée dans le code GENESIS est celle du CERC (HANSON et al., 1991) :

$Q=\left(H^{2} C_{g}\right)_{b}\left(a_{1} \sin \left(2 \alpha_{b s}\right)-a_{2} \cos \left(2 \alpha_{b s}\right) \frac{\partial H}{\partial x}\right)_{b}$

où $H$ : la hauteur de houle,

$C_{g}$ : la célérité de groupe de la houle en accord avec la théorie linéaire,

$b$ : l'indice dénotant la condition de houle déferlante,

$\alpha_{b s}$ : l'angle entre la crête de la vague au déferlement et le rivage,

$a_{1}=\frac{K_{1}}{16\left(\rho_{s} / \rho-1\right)(1-p) 1.416^{5 / 2}}$

$a_{2}=\frac{K_{2}}{8\left(\rho_{s} / \rho-1\right)(1-p) \tan (\beta) 1.416^{5 / 2}}$

où $K_{1}$ et $K_{2}$ sont deux paramètres du modèle, $\rho_{s}$ est la masse volumique du sédiment, $\rho$ la masse volumique de l'eau, $p$ la porosité du sédiment et enfin, $\tan (\beta)$ est la pente moyenne de la plage.

\subsection{Données d'entrée}

Les données utilisées pour la création du modèle sont :

- Les données bathymétriques issues de la numérisation et le géo-référencement des cartes marines des années 1996 et 2000 à l'échelle 1/5000 (sous la projection UTM Nord, fuseau 32, système géodésique WGS84).

- Les traits de côte des années 1996 et 2000.

- Les mesures de houle sur quatre ans, ces enregistrements comportent la date, l'heure, la hauteur significative $\left(H_{S}\right)$, la période $(T)$ et l'orientation $(\theta)$.

\subsection{Méthodologie pour la modélisation numérique}

La première étape a consisté en la création du maillage pour STWAVE et GENESIS comme le montre la figure 2 et l'insertion des stations pour la ligne de référence (position du trait de côte de l'année 1996) en utilisant le module GRIDGEN. Ces stations sont créées à ce stade pour le stockage des résultats de simulation par STWAVE.

La deuxième étape a été la préparation des données du modèle de propagation de houle. Il s'agit de transformer les données de houle avec le module WWWL, les classifier en catégories à l'aide de WSAV, comme le montre la figure 3, et enfin former des permutations selon l'ajustement spectral des évènements de la houle en utilisant le module SPECGEN.

L'étape suivante a consisté à simuler, à l'aide de STWAVE, la propagation de la houle jusqu'à l'emplacement des stations situées juste avant le déferlement et d'établir les caractéristiques des houles après leur propagation. 


\section{XIII ${ }^{\text {èmes }}$ Journées Nationales Génie Côtier - Génie Civil \\ Dunkerque, 2-4 juillet 2014}

La dernière étape a consisté à simuler l'évolution de la ligne de littoral avec le module GENESIS. Pour cela, on a dû simuler l'évolution du littoral entre les années 1996 et 2000 et ceci nous a permis d'une part d'effectuer le calage du modèle (les paramètres de transport $K_{1}$ et $K_{2}$, le paramètre de calage raffiné "angle offset" $\phi$ ) et, d'autre part, de procéder à des tests de sensibilité du modèle avec la variation du diamètre médian des grains de sable $d_{50}$ et la hauteur de la berme $d_{b}$.

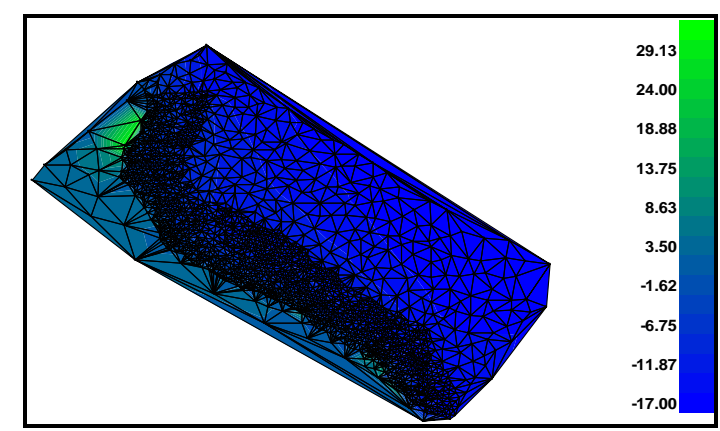

Figure 2. Maillage par Triangulation de la bathymétrie par GRIDGEN.

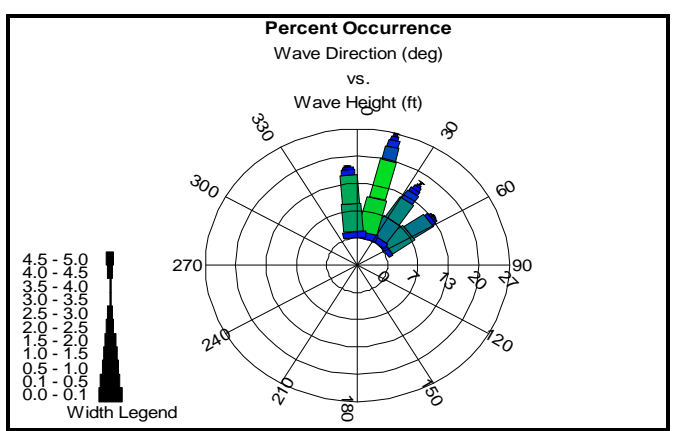

Figure 3. Rose de houle de pourcentage d'occurrence des houles.

\section{Résultats des simulations et discussion}

\subsection{Simulations de la propagation de la houle}

STWAVE est formulé sur une grille cartésienne, selon un système de coordonnées local. L'orientation de l'axe des $x\left( \pm 87,5^{\circ}\right)$ définit le demi-plan qui est représenté dans le modèle (RESIO et al., 2001). Les limites latérales du rivage sont définies en fonction des caractéristiques de la zone d'intérêt et les cellules de la grille sont carrées $\left(D_{\mathrm{x}}=D_{\mathrm{y}}\right)$. Le modèle de houle STWAVE, après simulation, permet alors de calculer les caractéristiques des houles au niveau des stations de la ligne de référence.

La figure 4 montre, suite à une coupe perpendiculaire au rivage qui passe par une station (exemple pour $Y=4250$ ), la profondeur de fermeture calculée qui correspond au point de la cassure de la courbe. La figure 5 montre le résultat des simulations de la propagation de la houle après correction des positions des stations.

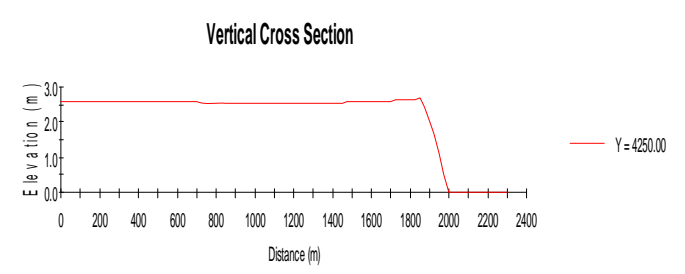

Figure 4. Profondeur de fermeture calculée.

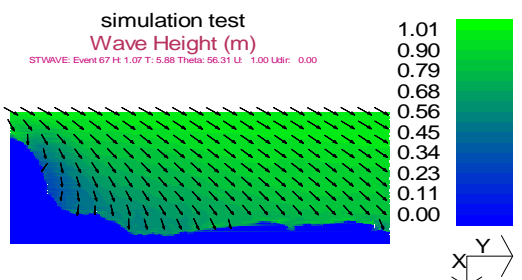

Figure 5. Résultats de la propagation de la houle par STWAVE. 


\section{Thème 2 - Dynamique sédimentaire}

\subsection{Simulations par GENESIS}

\subsubsection{Conditions aux limites latérales}

Le domaine d'étude est initialement situé entre deux caps (cap Gammarth et colline Sidi Bou Saïd). Pour la limite sud, on a choisi l'option "moving" avec une vitesse d'érosion de -3.5 m pendant la période de simulation et pour le côté nord, nous avons assimilé le cap à un épi "groin" dont la distance au rivage depuis son extrémité est relativement petite et donc permet le passage des sédiments.

\subsubsection{Calage du modèle}

Les paramètres du calage sont les variables de calage $K_{1}, K_{2}$ et l'angle de variation de la direction de propagation de la houle "angle offset" $\phi$. Le travail de calage consiste à définir les paramètres de réglage en simulant une évolution passée du trait de côte. En partant d'une position initiale du trait de côte (année 1996) on simule l'évolution de la côte pour l'année 2000 puis on entame une série de variations de ces paramètres en vue de choisir les valeurs les plus appropriées selon le degré d’adéquation obtenu entre la ligne de rivage cible et celle calculée par GENESIS pour l'année 2000. Les valeurs de $K_{1}, K_{2}$ et $\phi$ retenues sont $K_{1}=0.5, K_{2}=0.5$ et $\phi=-5^{\circ}$.

\subsubsection{Sensibilité aux paramètres du modèle}

Le test de sensibilité consiste à varier les données d'entrée de GENESIS. La variation du diamètre médian $\mathrm{d}_{50}$ des grains cause une variation peu significative à l'évolution du littoral mais le modèle est plus sensible à la variation de la hauteur de berme.

\subsubsection{Simulations de l'évolution de la ligne de côte}

Il s'agit de présenter les résultats de l'évolution du trait de côte jusqu’à l'année 2030 ainsi que différents plans d'action qui visent à protéger les zones du littoral les plus vulnérables à l'érosion. Pour cela des simulations ont été effectuées dans le but d'amener la configuration du littoral jusqu'à l'année actuelle (2014). D’après les simulations un volume d'échange annuel moyen égal à $41900 \mathrm{~m}^{3}$ a été enregistré entre les années 2000 et 2014.

\subsubsection{Evolution naturelle de la côte}

En partant de la position de la ligne de côte de l'année 2014, on tente de prédire l'évolution de la position de la ligne de côte durant les prochaines années.

L'idée est d'évaluer l'évolution de la ligne de côte dans la zone étudiée jusqu'à une position finale dans un avenir proche ainsi que lointain.

À partir de la figure 6 nous notons des zones qui subissent l'érosion : dans le secteur de Gammarth, la zone entre Borj Ben Ayed jusqu’à l’hôtel Movenpick est caractérisée par des espaces sableux peu développés. Dans le secteur de La Marsa, au-delà de la 


\section{XIII ${ }^{\text {èmes }}$ Journées Nationales Génie Côtier - Génie Civil \\ Dunkerque, 2-4 juillet 2014}

Coupole, on note une érosion faible de la plage. En effet, cette zone est protégée partiellement par la présence des platiers rocheux et sa localisation par rapport aux houles frontales. Des zones d'engraissement sont à l'inverse identifiées : au niveau de Gammarth, zone concave près de l'hôtel Movenpik et des parties limitées de la plage de Marsa Cube et la plage de Corniche.

Des zones stables, caractérisées par une évolution peu significative, sont localisées au niveau de plage de la Marsa. Celle-ci est due essentiellement d'une part, à la forme d'une anse qui épanouit l'énergie de la houle et d'autre part à la protection des deux caps (cap Gammarth et colline Sidi Bou Saïd). Ces résultats nous ont permis d'évaluer les zones les plus vulnérables à l'érosion et de préparer des plans d'action afin de les protéger.

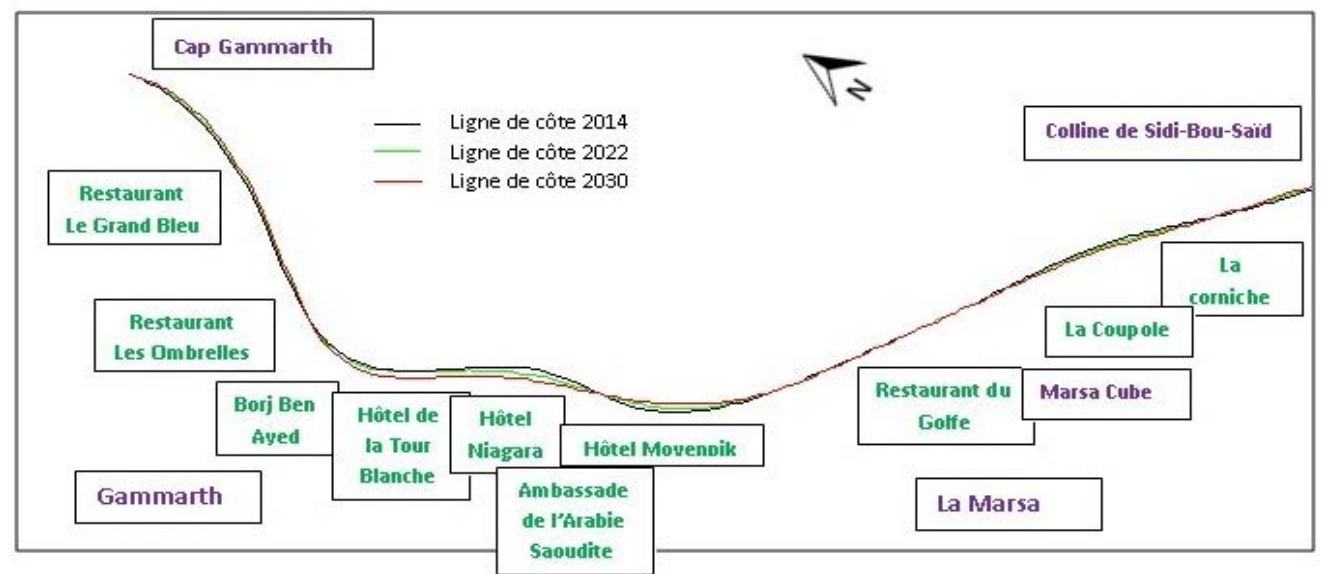

Figure 6. Résultats des simulations des années 2014, 2022 et 2030 (sortie de GENESIS).

\subsubsection{Simulations des scénarios des aménagements proposés}

Proposition 1 - L'emplacement des épis :

L'impact de l'implantation de deux épis est testé en premier lieu entre les années 2014 et 2018 pour la détermination des paramètres de dimensionnement des épis.

Après avoir fixé les paramètres de dimensionnement des épis (épi 1 de $200 \mathrm{~m}$ de longueur, épi 2 de $150 \mathrm{~m}$ de longueur, taux de sable passant à travers l'épi égal à 0.4 ), nous proposons d'effectuer une série de simulations pour évaluer l'effet des aménagements jusqu'à l'année 2030. La figure 7 montre la formation d'une plage qui est assurée par la présence des épis. Le volume cumulé par les épis, durant les premières huit années de simulation (figure 8), diminue en fonction du temps mais l'évolution de la zone d'érosion en aval est limitée ; cela est lié au choix de la valeur du taux de sable passant à travers l'épi (taux désigné "groin sand permeability" par HANSON, 1988). 


\section{Thème 2 - Dynamique sédimentaire}

Proposition 2 - Rechargement de plage protégée par un épi :

La deuxième proposition consiste à procéder à un rechargement artificiel pour restaurer la plage érodée. Un site de rechargement de 850 mètres de longueur est proposé, présenté dans la figure 9.

Pour l'épi de protection (l'épi est localisé à $1837 \mathrm{~m}$ au sud du Cap Gammarth), le taux de sable passant à travers l'épi est fixé à 0.3 et la longueur à $150 \mathrm{~m}$. Cette valeur permettra la transmission partielle des sédiments et surtout la limitation de l'effet de l'érosion en aval de l'épi. Le débit perdu sera compensé par des volumes d'entretien. Pour la simulation à long terme (jusqu'à l'année 2030), deux volumes d'entretien seront injectés dans le système pendant les années 2020 et 2028 avec une hauteur de berme estimée à $0.5 \mathrm{~m}$. La courbe "Final 2014" sur la figure 10 présente la courbe initiale de la simulation, l'effet de l'implantation de l'épi n’est visible alors qu’à partir de l'année 2018. Les évolutions du reste des courbes semblent très proches avec une tendance à l'engraissement.

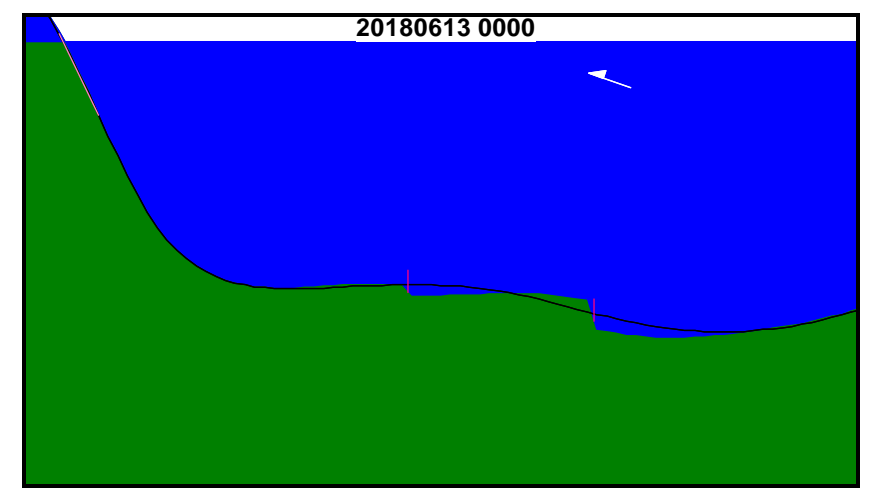

Figure 7. Trait de côte simulé après l'implantation des deux épis (année 2018).

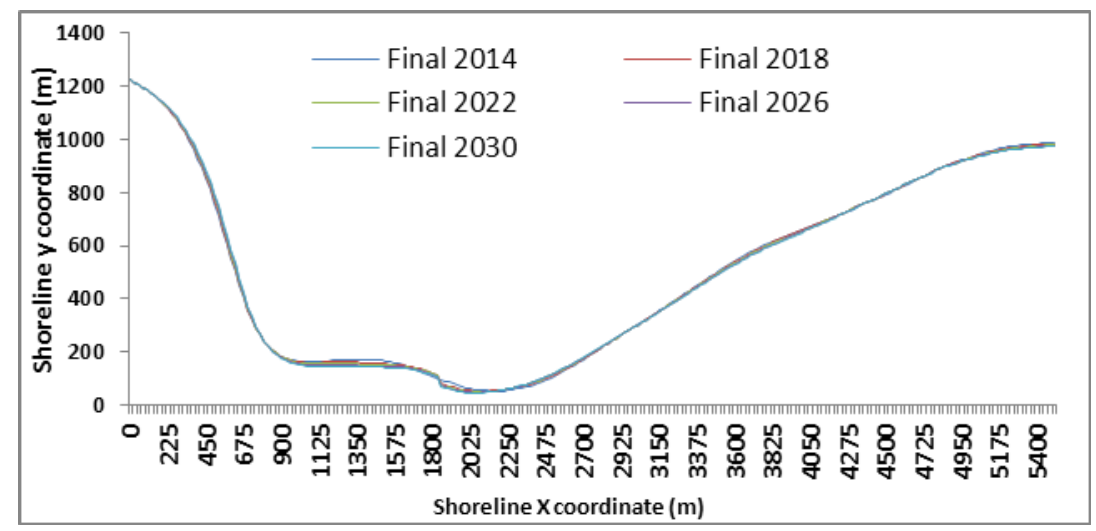

Figure 8. Evolution du trait de la côte après l'implantation des épis (Position de l'épi 1 : $x=1362$ m. Position de l'épi 2 : x=1837 m). 


\section{XIII ${ }^{\text {èmes }}$ Journées Nationales Génie Côtier - Génie Civil \\ Dunkerque, 2-4 juillet 2014}

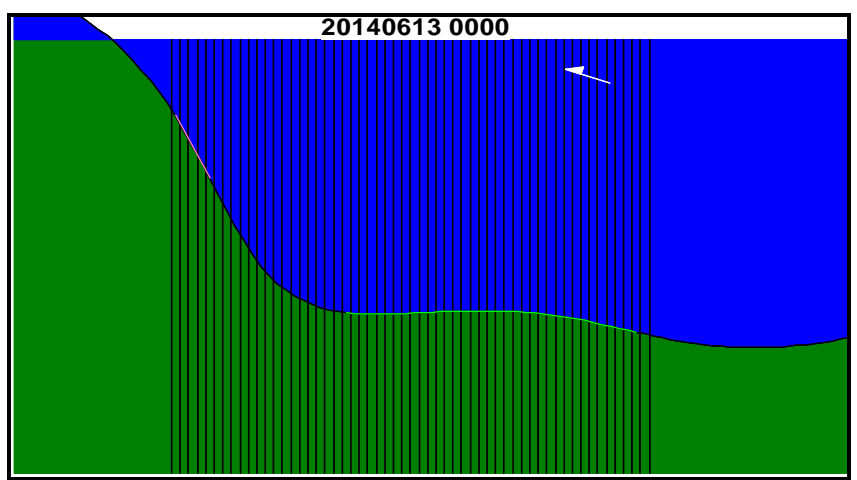

Figure 9. Placement de la zone de rechargement de plage (vert clair) dans la configuration GENESIS.

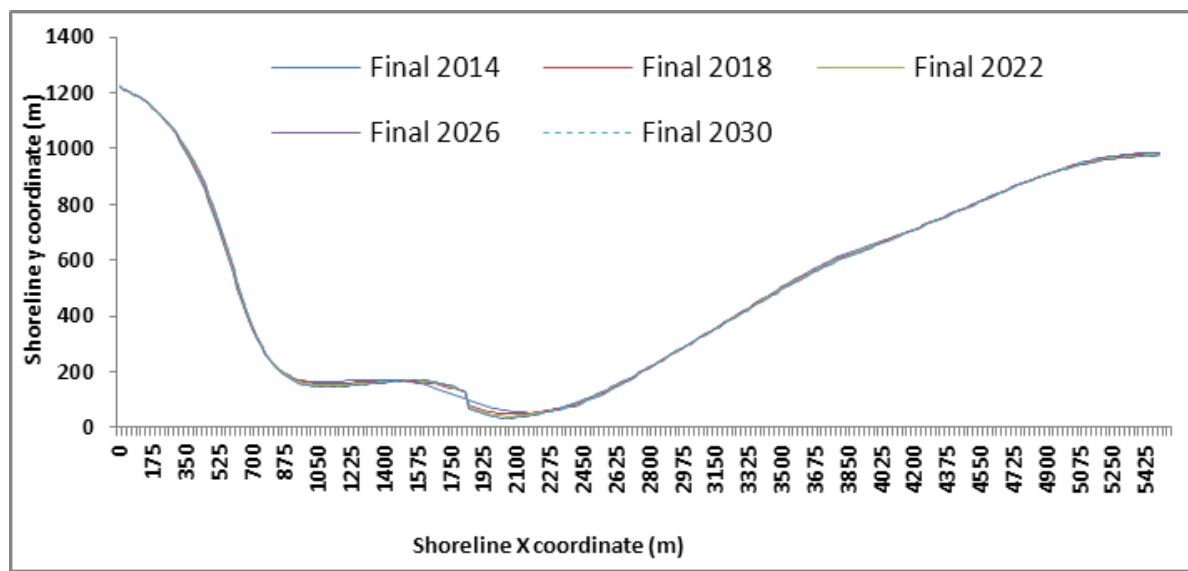

Figure 10. Evolution du littoral pour une plage rechargée et protégée par un épi.

\section{Conclusions}

Dans ce travail, nous nous sommes intéressées à la dynamique sédimentaire de la côte de la zone Gammarth-La Marsa, pour cela nous avons simulé la propagation de la houle à l'aide du module STWAVE puis nous avons entamé les simulations GENESIS et effectué les tests de sensibilité du modèle et le travail de calage. Nous avons ensuite modélisé l'évolution du littoral à partir de la ligne de côte observée de 1996 jusqu'à l'horizon 2030. Cette simulation nous a permis de localiser les zones de stabilisation, d'engraissement ainsi que la zone la plus touchée par l'érosion : il s'agit de la zone depuis Borj Ben Ayed jusqu'au rivage de l'hôtel Movenpick. Ces résultats nous ont poussé à étudier des scénarios de protection de la zone en question pour effectuer par la suite des séries de simulations dans le but de proposer la méthode d'intervention la plus efficace. D’après les résultats des simulations, nous avons privilégié la solution de rechargement de plage avec une protection par un épi d'une longueur de $150 \mathrm{~m}$. Le modèle élaboré nous a permis d'obtenir des résultats satisfaisants quant à l'avancement ou le recul de la côte étudiée. Cependant, GENESIS présente des limitations du fait qu'il est de type "one-line", cela suppose que la forme du profil de plage reste 
inchangée. Ainsi ce profil se déplace parallèlement à lui-même, vers le rivage (érosion) ou au large (engraissement) sans changer de forme.

\section{Références bibliographiques}

APAL (2003). Agence de Protection et d'Aménagement du Littoral. Rapport d'Etude Générale pour la Protection du Littoral Tunisien.

HANSON H. (1988). GENESIS-A generalized shoreline change numerical model. Journal of Coastal Research, Vol. 5(1), pp 1-27.

HANSON H., GRAVENS M.B., KRAUS N.C. (1991). GENESIS : Generalized model for simulating shoreline change. Report $\mathrm{n}^{\circ} 2$, Workbook and system user's manual, U.S. Army Corps of Engineers, Coastal Engineering Research Center, USA

RESIO D.T., SMITH J.M., SHERLOCK A.R. (2001). Coastal and Hydraulics Laboratory. U.S. Army Engineer Research and Development Center Webographie. 